\title{
Management of Psoriasis With Biologics in Clinical Practice: An Update for 2020
}

\author{
Mirjana G. Ivanic, BA; Sheida Naderi-Azad, BS; Shikha Walia, BS; George Han, MD, PhD; \\ Jashin J. Wu, MD
}

\section{PRACTICE POINTS}

- Inform patients about current data guiding treatment from clinical trials of biologics.

- Explain to patients that finding the treatment that is the best fit for them may require trial and error, as everyone responds to treatments differently.

- Consult with patients about misconceptions and potential fears about biologics and what the protocol is for monitoring safety during treatment.
This article aims to address updates on recent clinical trial findings (April 2019 to April 2020) regarding biologic therapy initiation and maintenance for adult patients. Prescribers should use this update as guidance for determining the appropriate biologic class based on patient characteristics and for approaching biologicexperienced patients with refractory psoriasis. This update also may serve as a reference for the recommended dosing regimens of the 11 approved biologics.

Cutis. 2020;106(suppl 2):21-24.

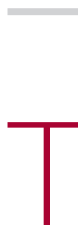

he advent of biologic therapy over the last 2 decades has transformed the treatment of psoriasis; patients who either are not good candidates for or have an inadequate response to traditional treatments (topicals and/or phototherapy) now have numerous options for treatment. ${ }^{1}$ Patients burdened by extensive disease, recurrent flares, and stubborn treatment areas are ideal candidates for biologics. There are 11 biologics approved by the US Food and Drug Administration (FDA) (Table) for treating moderate to severe plaque psoriasis as supported by grade A evidence. The FDA has authorized 1 new biologic_risankizumab-since the joint guidelines from the American Academy of Dermatology and National Psoriasis Foundation were released for the treatment of psoriasis with biologics. ${ }^{2}$ This article aims to address updates on recent clinical trial findings (April 2019 to April 2020) regarding biologic therapy initiation and maintenance for adult patients. Prescribers should use this update as guidance for determining the appropriate biologic class based on patient characteristics and for approaching biologic-experienced patients with refractory psoriasis. This update also may serve as a reference for the recommended dosing regimens of the 11 approved biologics.

\section{Using Risankizumab}

Risankizumab is a new biologic that selectively targets the IL-23 pathway by binding the p19 subunit of IL-23. It was approved by the FDA in April 2019. Two recent studies have demonstrated the efficacy of risankizumab in disease management. ${ }^{3,4}$

\footnotetext{
Ms. Ivanic is from Meharry Medical College, Nashville, Tennessee. Ms. Naderi-Azad is from the University of Toronto Faculty of Medicine, Ontario, Canada. Ms. Walia is from Lake Erie College of Osteopathic Medicine, Pennsylvania. Dr. Han is from the Icahn School of Medicine at Mount Sinai, New York, New York. Dr. Wu is from the Dermatology Research and Education Foundation, Irvine, California.

Ms. Ivanic, Ms. Naderi-Azad, and Ms. Walia report no conflict of interest. Dr. Han is or has been a consultant/advisor, investigator, or speaker for AbbVie; Athenex; Boehringer Ingelheim; Bond Avillion; Bristol-Myers Squibb; Celgene Corporation; Eli Lilly and Company; Janssen Biotech, Inc; LEO Pharma; MC2 Therapeutics; Novartis; Ortho Dermatologics; PellePharm; Pfizer; Regeneron Pharmaceuticals, Inc; Sanofi Genzyme; Sun Pharmaceutical; and UCB. Dr. Wu is or has been a consultant, investigator, or speaker for AbbVie; Almirall; Amgen; Arcutis; Boehringer Ingelheim; Bristol-Myers Squibb; Celgene Corporation; Dermavant; Dermira; Dr. Reddy's Laboratories; Eli Lilly and Company; Janssen Biotech, Inc; LEO Pharma; Novartis; Regeneron Pharmaceuticals, Inc; Sanofi Genzyme; Sun Pharmaceutical; UCB; and Valeant Pharmaceuticals North America. Correspondence: Jashin J. Wu, MD (jashinwu@gmail.com). doi:10.12788/cutis.0035
} 


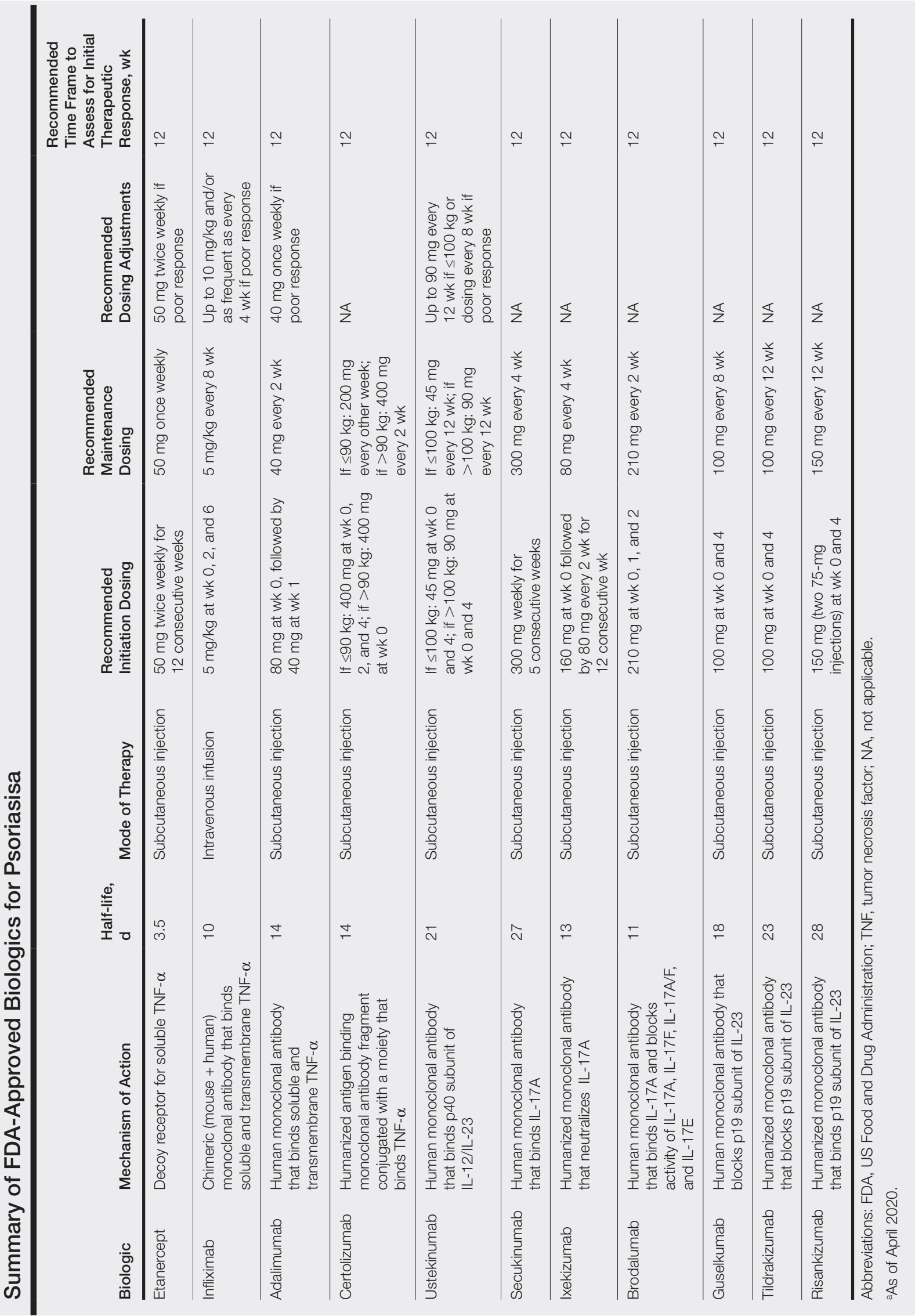


IMMvent was a double-blind, 2-part, phase 3, randomized controlled trial (RCT) of participants 18 years and older $(\mathrm{N}=605)$ with moderate to severe psoriasis (with or without psoriatic arthritis) across 11 countries. $^{3}$ Inclusion criteria consisted of psoriasis involving at least $10 \%$ of the body surface area (BSA), absolute psoriasis area and severity index (PASI) score of 12 or higher, and static physician global assessment (sPGA) score of 3 or higher. Prior biologic treatment did not preclude study entry (excluding risankizumab or adalimumab), and nearly $40 \%$ of participants previously had been on a different biologic. Notably, this trial allowed for inclusion of patients with prior malignancy ( $>5$ years prior) and patients who tested positive for exposure to tuberculosis (TB) but were not shown to have active TB (provided appropriate treatment for latent TB was started). Study participants identified as white (81\%), Asian (14\%), black/African American (4\%), or other ethnicity (1\%). Part A involved administration of $150 \mathrm{mg}$ risankizumab $(n=301)$ at weeks 0 and 4 or $80 \mathrm{mg}$ adalimumab $(n=304)$ loading dose at week 0 followed by $40 \mathrm{mg}$ at week 1 and $40 \mathrm{mg}$ every other week thereafter until the end of week 15. At week 16 there was a significant difference in proportion of participants achieving $90 \%$ or more improvement (PASI-90) with risankizumab (72\%) vs adalimumab $(47 \%)(P<.0001)$ and achieving an sPGA score of 0 or 1 (clear or almost clear) with risankizumab $(84 \%)$ vs adalimumab $(60 \%)(P<.0001)$. In part B (weeks 16-44), adalimumab immediate responder (PASI $\geq 50$ to PASI <90) participants were re-randomized to continue adalimumab $40 \mathrm{mg}$ every other week (starting from week 17 and stopping at week 44) or switch to $150 \mathrm{mg}$ risankizumab administered at weeks 16, 20, and 32 . Patients taking risankizumab in part A continued the drug, administered at weeks 16 and 28. At week 44, there was a significant difference in percentage of participants achieving PASI-90 with risankizumab (66\%) vs adalimumab $(21 \%)(P<.0001){ }^{3}$

IMMhance was another double-blind phase 3 RCT with 2 parts that assessed the clinical efficacy of risankizumab compared to placebo in patients 18 years or older $(\mathrm{N}=507)$ across 9 countries with the same inclusion criteria for patients as IMMvent. ${ }^{4}$ Part A involved administration of $150 \mathrm{mg}$ risankizumab $(n=407)$ or placebo $(n=100)$ at weeks 0 and 4 using a 4:1 random allocation ratio. At week 16, regardless of initial treatment, all participants received $150 \mathrm{mg}$ risankizumab. Treatment results at week 16 showed a significant difference in percentage of participants achieving PASI-90 with risankizumab (73.2\%) vs placebo $(2.0 \%)(P<.001)$ and sPGA score of 0 or 1 with risankizumab $(83.5 \%)$ vs placebo $(7.0 \%)(P<.001)$. Furthermore, in part B (weeks 16-104), at week 28 participants on risankizumab with an sPGA score of 0 or 1 were randomized with a 1:2 allocation ratio to continue $150 \mathrm{mg}$ risankizumab or switch to placebo to produce a treatment withdrawal effect. Part B results showed a significant difference in the proportion of participants achieving an sPGA score of 0 or 1 with risankizumab $(87.4 \%)$ vs placebo $(61.3 \%)(P<.001)$ at week 52 and at week 104 with risankizumab (81.1\%) vs placebo (7.1\%) $(P<.001)$. Risankizumab was well tolerated, with the most common adverse events (AEs) being nasopharyngitis (23.4\%), upper respiratory tract infection (15.4\%), and headache (6.8\%). Serious AEs included cancer $(2.6 \%$; 2.2 events per 100 patient-years), hepatic events (4.6\%) including hepatic cirrhosis $(0.2 \%)$, and serious infections (1.8\%; 1.4 events per 100 patient-years). ${ }^{4}$

Overall, the strengths of risankizumab with regard to its clinical efficacy and utility in biologic-experienced patients were confirmed in these studies. The inclusion of patients with prior treated malignancy and positive $\mathrm{TB}$ tests also was more in line with what one might encounter with real-world practice and, as such, provided valuable data to help aid treatment decisions. These 2 studies provided valuable evidence for the therapeutic benefit and relatively mild safety profile of risankizumab in treatment of moderate to severe psoriasis for patients with and without prior biologic therapy.

\section{Choosing a Biologic}

Refractory psoriasis involves nonresponse (primary failure) or return of disease symptoms after initial improvement (secondary failure) with a biologic. Selecting a biologic for patients who have experienced prior biologic failure is difficult. It is still unknown whether it is more efficacious for patients to try a same-class drug or a biologic targeting a different inflammatory pathway or cytokine. Studies have shown mixed results regarding how to manage patients with biologic failure, with both approaches demonstrating positive outcomes.

One analysis of the Corrona Psoriasis Registry included 144 patients, the majority of whom (89.8\%) were biologic experienced, who began secukinumab treatment and returned for a 6-month follow-up (5-9 months). ${ }^{5}$ Patients enrolled in the registry were 18 years or older, had been diagnosed with psoriasis by a dermatologist, and initiated or switched an FDAapproved systemic agent or biologic within the last 12 months. Of biologic-experienced participants, 37.7\% had used 3 or more biologics. More than half of included participants were either male $(55 \%)$ or obese $(53.4 \%)$. Comorbidities included hypertension (43.2\%), hyperlipidemia $(33.9 \%)$, anxiety $(20.3 \%)$, diabetes mellitus $(15.3 \%)$, cardiovascular disease $(14.4 \%)$, and depression $(13.6 \%)$. After 6 months of treatment, there was significant improvement in the involvement of BSA (mean difference, -12.1), investigator global assessment score $(-1.5)$, dermatology life quality index (DLQI) $(-4.8)$, pain $(-23.2)$, itch $(-30.8)$, fatigue $(-8.8)$, and work productivity $(-9.2)(P<.01)$. Secukinumab therapy displayed notable reduction in symptom severity in this population with difficult-to-treat psoriasis. Its relative success in this cohort provides support for its use in treating patients who have failed other classes of biologics. ${ }^{5}$ 
Evidence supporting reduction of pruritus and pain with secukinumab also was notable. The CLEAR phase 3 RCT involved participants treated with $300 \mathrm{mg}$ secukinumab every week for the first 4 weeks and then every 4 weeks thereafter for 48 weeks $(n=312)$, up to 100 weeks $(n=277) .6$ Participants had complete relief of pain (score 0), itching, and scaling at week 16 (69.4\%, $49.7 \%$, and $61.2 \%$, respectively), week 52 (67.1\%, 48.9\%, and 53.3\%, respectively), and week 104 (70.9\%, 47.4\%, and $54.8 \%$, respectively). Reported AEs included candida infections $(7.2 \%)$, malignant or unspecified tumors $(1.5 \%)$, and neutropenia $(<1 \%){ }^{6}$

Researchers investigated intraclass switching to brodalumab with prior failure of IL-17 inhibitors. An openlabel study involved participants $(n=39)$ with prior failure with secukinumab or ixekizumab therapy. ${ }^{7}$ Participants were administered $210 \mathrm{mg}$ brodalumab with standard dosing at weeks 0,1 , and 2 , and then every 2 weeks thereafter. At week 16, 69\% of participants achieved PASI-75, $44 \%$ achieved PASI-90, 28\% achieved PASI-100, and 62\% achieved an sPGA score of 0 or 1 . The authors attributed the relative success of brodalumab compared to prior anti-IL-17 agents to inhibition of the IL-17 receptor with brodalumab rather than the IL-17A ligand. ${ }^{7}$ Brodalumab may be a useful alternative biologic for patients with nonresponse to and secondary failure with biologics, including the IL-17A inhibitors.

Recent findings support effective skin clearance and improved symptom management with ixekizumab and ustekinumab. Of note, ixekizumab was reported to provide rapid improvement in skin lesions and quality of life to a greater extent than guselkumab.

The IXORA-R double-blinded RCT compared the clinical benefit of participants 18 years and older taking standard approved dosages of ixekizumab $(n=520)$ or guselkumab $(n=507){ }^{8}$ Patients were included if they had plaque psoriasis for at least 6 months before baseline, an sPGA score of at least 3, PASI score of 12 or higher, $10 \%$ or greater BSA, no prior IL-17 inhibitor failure, no use of IL-23 p19 inhibitors, and no use of any biologic within the specified period prior to baseline. At week 12, ixekizumab showed superior clinical improvement measured by the proportion of participants achieving complete skin clearance (ie, PASI-100) $(41 \%)$ compared to guselkumab $(25 \%)(P<.001)$. There were more participants taking ixekizumab who reported DLQI of 0 or 1 (no impact of disease on quality of life) $(34 \%)$ compared to guselkumab $(21 \%)(P<.001)$ as early on as week 4 . The most common AE was upper respiratory tract infection $(7 \%)$ in both groups. The risk of treatment-emergent AEs (56\%), discontinuation because of AEs (2\%), and serious AEs (3\%) were comparable in both groups. The number of injection-site reactions was higher with ixekizumab (13\%) vs guselkumab (3\%). The authors concluded that ixekizumab offers the ability to provide rapid relief of symptoms, which is associated with improved DLQI. ${ }^{8}$
Response to ustekinumab therapy was assessed in a patient cohort enrolled in the Corrona Psoriasis Registry. This study involved 178 participants 18 years and older with psoriasis involvement of $3 \%$ or greater BSA who were treated with ustekinumab. ${ }^{9}$ By their 6-month follow-up visit, $55.6 \%$ of participants achieved adequate treatment response (BSA improving to $<3 \%$ or $75 \%$ from enrollment). Increasing patient age was significantly associated with decreased likelihood of achieving a response (odds ratio, 0.981 [95\% confidence interval, $0.962-0.999] ; P=.049)$. Ustekinumab is a practical option for psoriasis treatment that seems to yield better results in younger patients. ${ }^{9}$ This evidence reveals that increased patient age is a characteristic that may contribute to poor treatment response and should be considered when choosing the best fit for biologic therapy.

\section{Final Thoughts}

Using evidence-based interventions to treat patients is the cornerstone of ethical and high-quality medical care. This guide sought to provide relevant updates in a variety of both comparator and pivotal trials, with the goal of summarizing clinically relevant information that may be extracted from these trials to guide patient care. It is not an exhaustive review but may be utilized as a reference tool to fine-tune selection criteria in choosing 1 of 11 biologics for the treatment of psoriasis.

\section{REFERENCES}

1. Pithadia DJ, Reynolds KA, Lee EB, et al. Translating the 2019 AAD-NPF Guidelines of Care for the Management of Psoriasis With Biologics to clinical practice. Cutis. 2019;104(suppl 2):12-16.

2. Menter A, Strober BE, Kaplan DH, et al. Joint AAD-NPF guidelines of care for the management and treatment of psoriasis with biologics [published online February 13, 2019]. J Am Acad Dermatol. 2019;80:1029-1072.

3. Reich K, Gooderham M, Thaçi D, et al. Risankizumab compared with adalimumab in patients with moderate-to-severe plaque psoriasis (IMMvent): a randomised, double-blind, active-comparator-controlled phase 3 trial. Lancet. 2019;394:576-586.

4. Blauvelt A, Leonardi CL, Gooderham M, et al. Efficacy and safety of continuous risankizumab therapy vs treatment withdrawal in patients with moderate to severe plaque psoriasis: a phase 3 randomized clinical trial. JAMA Dermatol. 2020;156:649-658.

5. Strober BE, Germino R, Guana A, et al. US real-world effectiveness of secukinumab for the treatment of psoriasis: 6-month analysis from the Corrona Psoriasis Registry. J Dermatolog Treat. 2020;31:333-341.

6. Thaçi D, Puig L, Reich K, et al. Secukinumab demonstrates sustained efficacy in clearing skin and improving patient-reported outcomes in patients with moderate-to-severe psoriasis through 2 years of treatment: results from the CLEAR study. J Am Acad Dermatol. 2019;81:1405-1409.

7. Kimmel G, Chima M, Kim HJ, et al. Brodalumab in the treatment of moderate to severe psoriasis in patients when previous anti-interleukin 17A therapies have failed. J Am Acad Dermatol. 2019;81:857-859.

8. Blauvelt A, Papp K, Gottlieb A, et al. A head-to-head comparison of ixekizumab vs. guselkumab in patients with moderate-to-severe plaque psoriasis: 12-week efficacy, safety and speed of response from a randomized, double-blinded trial. Br J Dermatol. 2020;182:1348-1358.

9. Van Voorhees AS, Mason MA, Harrold LR, et al. Characterization of insufficient responders to ustekinumab in patients with moderateto-severe psoriasis in the US Corrona Psoriasis Registry [published online February 27, 2020]. J Dermatolog Treat. doi:10.1080/09546634. 2020.1720586 . 